\title{
Interação Humano Computador e o Grupo de Pesquisa em Informática na Educação da UDESC
}

\author{
Isabela Gasparini \\ Departamento de Ciência de \\ Computação \\ Universidade do Estado de Santa \\ Catarina (UDESC) \\ isabela.gasparini@udesc.br
}

\author{
Avanilde Kemczinski \\ Departamento de Ciência de \\ Computação \\ Universidade do Estado de Santa \\ Catarina (UDESC) \\ avanilde.kemczinski@udesc.br
}

\author{
Rebeca Schroeder \\ Departamento de Ciência de \\ Computação \\ Universidade do Estado de Santa \\ Catarina (UDESC) \\ rebeca.schroeder@udesc.br
}

\begin{abstract}
RESUMO
O Grupo de Pesquisa em Informática na Educação (GPIE) está localizado no Departamento de Ciência da Computação - DCC, do Centro de Ciências Tecnológicas - CCT, da Universidade do Estado de Santa Catarina - UDESC - Joinville desde 2002 e é certificado pela instituição e pelo CNPq. As investigações do grupo estão relacionadas ao uso e desenvolvimento de Tecnologias de Informação e Comunicação - TIC, como elemento facilitador do processo ensino-aprendizagem presencial, semipresencial e a distância. Este artigo apresenta algumas ações desenvolvidas no grupo, relacionadas à área de Interação Humano-Computador.
\end{abstract}

\section{PALAVRAS-CHAVE}

Grupo de pesquisa, GPIE, IHC, UDESC.

\section{INTRODUÇÃO}

O GPIE [1] é um grupo interdisciplinar que tem como linhas de pesquisa: (a) Ensino-Aprendizagem auxiliado por Computador e (b) Tecnologia Educacional. O GPIE atua junto aos estudantes dos cursos de graduação em Bacharelado em Ciência da Computação (BCC) e Tecnologia em Análise e Desenvolvimento de Sistemas (TADS) e nos programas de pós-graduação Programa de PósGraduação em Computação Aplicada (PPGCA) e Programa de PósGraduação em Ensino de Ciências, Matemática e Tecnologia (PPGECMT).

Dentro do GPIE os pesquisadores e estudantes atuam na pesquisa, por meio de projetos de pesquisa e de iniciação científica, em projetos de ensino e extensão para a comunidade, e estágios e desenvolvimento de produtos.

Os principais pesquisadores professores atuantes no grupo são: Prof ${ }^{a}$. Dra. Avanilde Kemczinski, Prof a Dra. Isabela Gasparini, Prof. Dr. Kariston Pereira, Prof. Dr. Adilson Vahldick, Prof. MSc. Alexandre Veloso de Matos e Prof ${ }^{\mathrm{a}}$ MSc. Luciana Rita Guedes.

\footnotetext{
Permission to reproduce or distribute, in whole or in part, material extracted from this work, verbatim, adapted or remixed, as well as the creation or production from the content of such work, is granted without fee for non-commercial use, provided that the original work is properly credited.

IHC 2019 - TRILHA FÓRUM DOS GRUPOS DE PESQUISA, Outubro 21-25, 2019, Vitória, Brasil. In Anais Estendidos do XVIII Simpósio Brasileiro sobre Fatores Humanos em Sistemas Computacionais. Porto Alegre: SBC.

(C) 2019 by the author(s), in accordance with the terms of the Creative Commons Attribution-NonCommercial 4.0 International Public License (CC BY-NC 4.0).
}

Além destes professores, há professores parceiros da mesma instituição, como a Prof ${ }^{a}$. Rebeca Schroeder, e de outras instituições, nacionais e internacionais, tais como a UTFPR, UFPR, UFRGS, UFSM, PUCRS, PUC-Rio, USP e a Universidade de Málaga (Espanha). Este artigo apresenta algumas das ações do grupo, especialmente relacionadas à área de Interação HumanoComputador.

\section{PROJETOS RELACIONADOS E FOMENTOS RECEBIDOS}

Nesta seção apresentamos alguns projetos. O primeiro tem como título Gamificação em Sistemas Adaptativos Educacionais. Esse projeto tem como objetivo entender como a gamificação pode auxiliar os estudantes no processo de ensino-aprendizagem, através da análise das técnicas relacionadas a cada perfil de usuário do sistema. Esta pesquisa criou um framework para auxiliar no design, desenvolvimento e avaliação da gamificação centrada no usuário. Mais informação em [2], [3]. Atualmente o projeto avança para buscar entender como a gamificação pode ser adaptada a diferentes perfis de usuários.

O projeto intitulado Ambientes Inteligentes Educacionais com Integração de Técnicas Learning Analytics e Gamificação é um projeto com fomento externo da FAPESC - Fundação de Amparo à Pesquisa e Inovação do Estado de Santa Catarina e visa utilizar técnicas de gamificação e de Learning Analytics em Ambientes Inteligentes Educacionais, para aumentar a motivação e engajamento do aluno, melhorando também sua interação e desempenho no ambiente educacional.

Uma pesquisa mais recente do grupo envolve a criação de um Modelo de autoria de exercícios para ambientes e-learning que envolve a abordagem inquiry-based learning e a web social. A ideia é diminuir a carga de trabalho dos professores e ao mesmo tempo fazer com que estudantes sejam mais ativos no processo de ensino-aprendizagem, fazendo que eles criem novos exercícios e que estes exercícios possam ser avaliados por outros estudantes.

Outra iniciativa está relacionada aos Sistemas de Recomendação, que são técnicas de software que fornecem sugestões de itens a serem recomendados para um usuário. As sugestões visam ajudar os usuários em vários processos de tomada de decisão. Há diversos estudantes do grupo trabalhando neste tema. Um dos aspectos estudados está relacionado a recomendar locais para publicações de artigos científicos, como base em mecanismos de busca científica 
heterogêneos. Outra iniciativa relacionada ao tema de Sistemas de Recomendação é entender como a apresentação da recomendação pode influenciar o comportamento do usuário, e quais as melhores práticas para a recomendação em sistemas educacionais [6].

Com o foco em apoiar comunidades acadêmicas, o projeto intitulado Redes Sociais Profissionais: extração e análise de dados tem coletado dados de veículos de publicação científicas para efetuar diversos tipos de análise sobre a rede de colaboração dos pesquisadores. Um dos trabalhos que atuou no contexto deste projeto coletou e analisou a rede de colaboração dos pesquisadores do Simpósio Brasileiro sobre Fatores Humanos em Sistemas Computacionais [4] (Figura 1). Além de dados de produtividade, este trabalho analisou a influência exercida por autores prolíficos deste simpósio ao longo dos anos. Os resultados produzidos até o momento permitiram expandir a compreensão da comunidade de pesquisadores do IHC, bem como identificar a formação de grupos e a iteração entre eles.

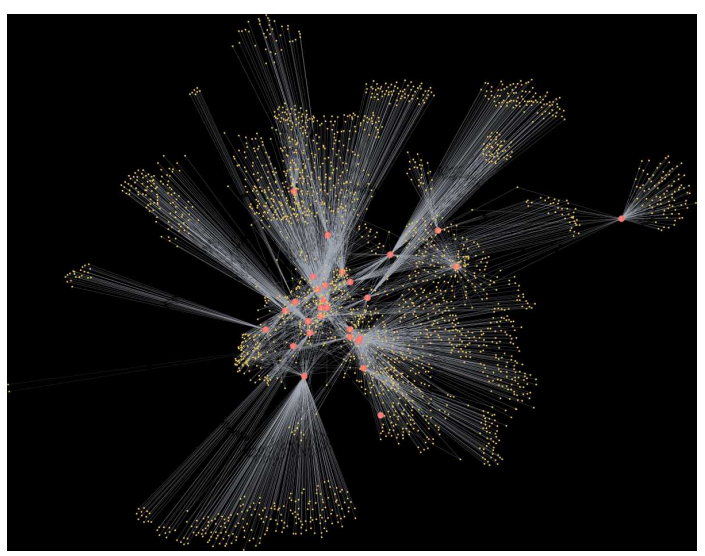

Figura 1: Rede de Coautoria do Simpósio IHC

A linha de Pensamento Computacional e Computação Desplugada tem atuado junto a professores e estudantes do ensino fundamental e médio. As ações envolvem oficinas e dinâmicas em sala de aula, com e sem o suporte de tecnologias. O uso de termos de consentimentos, questionários e entrevistas auxiliam nas ações planejadas. Além disso o projeto das experiências faz uso das técnicas de IHC, entendendo as necessidades dos usuários, projetando as atividades e realizando os experimentos.

O trabalho de Trajetórias de Aprendizagem busca entender as trajetórias dos estudantes em ambientes e-learning e analisar como o comportamento do usuário afeta os caminhos e interações em ambiente educacionais. Um trabalho futuro está relacionado a buscar entender quais características do usuário e de seu comportamento nos ambientes educacionais podem dar indícios e auxiliar a prever evasão em cursos online.

Além dos projetos e trabalhos de pesquisa citados, há um programa de extensão ligado à área de IHC, chamado Programa Interagir: Integração entre Ciência, Computação e Sociedade que teve início em janeiro de 2018. O programa possui dois eixos principais relacionados: o primeiro visa disseminar a Ciência e a Computação, e incentivar profissionais, professores e estudantes das áreas relacionadas (e.g. computação, matemática, engenharias, tecnologias, educação, etc.) a aprofundarem seus conhecimentos e habilidades. O segundo visa promover o nome da UDESC, enquanto instituição de ensino, pesquisa e extensão de qualidade, apta a contribuir com o desenvolvimento social, científico e tecnológico. Os eixos são apoiados por 4 projetos que englobam diversas ações, detalhados a seguir.

O projeto 'Eu quero programar' visa o Ensino de Algoritmos e Programação para a comunidade, que de um lado faz a conexão entre ensino-extensão, onde estudantes estão envolvidos na discussão da temática, visando a melhoria dos ambientes computacionais com a sociedade por meio de desenvolvimento tecnológico; e que de outro lado, compreende as questões técnicas para a realização de minicursos e planejamento de materiais para oferta destes à comunidade.

O projeto 'Catarinas' trata de ações afirmativas para envolvimento das mulheres na ciência, fortalecer e incentivar a presença da mulher na tecnologia por meio de oficinas, encontros e debates, abertos a comunidade sempre promovendo a igualdade e a inclusão.

O projeto 'Cientistas Anônimos' visa auxiliar estudantes a fazer ciência, apoiar a apresentação a produção científica e Internacionalização da universidade. Faz a conexão da pesquisaextensão de maneira que estudantes possam aprimorar suas técnicas de apresentação e comunicação, fazendo a difusão informacional da UDESC na comunidade. Além disso o projeto trata de ações sociais e motivacionais, apoiando estudantes em suas vivências acadêmicas, fazendo sua maior integração na universidade e com a comunidade local e regional.

O projeto LINGUESC - Centro de Idiomas do CCT-UDESC cria um ambiente dentro da universidade e que voluntários criam materiais e ministram aulas para estudantes e para a comunidade, apoiando a melhoria de outros idiomas. Todo o trabalho é voluntário, e as aulas são ministradas na universidade.

\section{AppIHC}

Uma solução desenvolvida pelo grupo é AppIHC [5], um aplicativo para auxiliar os participantes de eventos científicos a obterem informações sobre a programação do evento. Mais especificamente ele foi utilizado em 2017 e 2018 no Simpósio Brasileiro sobre Fatores Humanos em Sistemas Computacionais em 2017 e em 2018. O AppIHC faz uso de técnicas de gamificação e com Sistema de Recomendação embutido, recomendando as melhores sessões para os participantes do evento com base em diferentes informações (Figura 2). 
Interação Humano Computador e o Grupo de Pesquisa em Informática na Educação da UDESC

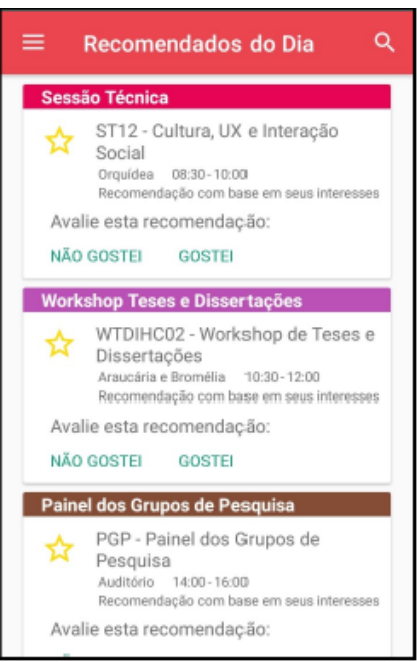

2018

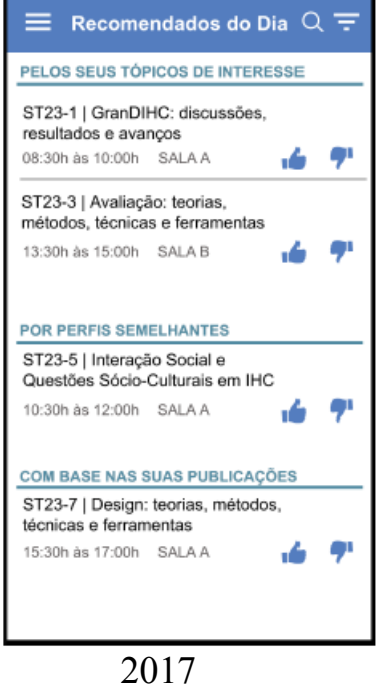

2017
Figura 2: Telas do AppIHC sobre as sessões recomendadas do dia em 2018 e 2017

\section{PRINCIPAIS OPORTUNIDADES E DESAFIOS}

Algumas oportunidades de pesquisa estão sendo exploradas no contexto do projeto relacionado a Redes Sociais Profissionais, sobretudo no que tange a rede de colaboração do Simpósio IHC e dos pesquisadores da comunidade. Um dos trabalhos em andamento considera os aspectos temporais das colaborações para compreender os mecanismos pelos quais a rede evolui. Deste modo, uma vez identificado o modelo de evolução, será possível prever ou recomendar novas parcerias de produção para os anos futuros deste simpósio.

Um outro trabalho no contexto deste projeto está analisando aspectos textuais de resumos de artigos em elaboração, para então recomendar veículos de publicações apropriados para a submissão. Para habilitar este tipo de análise, o desafio é coletar e integrar dados de diferentes veículos de publicação para comparar e relacionar com os dados do artigo para o qual se deseja a recomendação. Considerando a quantidade de fontes de dados que indexam estas informações, bem como o volume de dados associado, um dos problemas relevantes é determinar como se dará o gerenciamento destes dados. Além disso, será importante entender o perfil do usuário para recomendar de forma mais personalizada, e de acordo com as preferências individuais.

\section{AGRADECIMENTOS}

Agradecemos ao apoio financeiro da FAPESC, Edital chamada pública FAPESC/CNPQ No 06/2016 apoio a infraestrutura de CTI para jovens pesquisadores, projeto T.O. $\mathrm{N}^{\mathrm{o}}: 2017 \mathrm{TR} 1755$ Ambientes Inteligentes Educacionais com Integração de Técnicas Learning Analytics e Gamificação. O presente trabalho também foi realizado com apoio da UDESC. O presente trabalho foi realizado com apoio da Coordenação de Aperfeiçoamento de Pessoal de Nível Superior - Brasil (CAPES) - Código de Financiamento 001

\section{REFERÊNCIAS}

[1] Diretório de grupos de pesquisa do CNPq. GPIE/UDESC. Disponível em: <dgp.cnpq.br/dgp/espelhogrupo/7766290030234591>

[2] A. Klock, I. Gasparini, M. S. Pimenta. User-centered gamification for e-learning systems: a quantitative and qualitative analysis of its application. INTERACTING WITH COMPUTERS (to appear).

[3] A. Klock, I. Gasparini, M. S. Pimenta. Framework 5W2H: um guia para projetar, desenvolver e avaliar uma gamificação centrada no usuário. In: XV Brazilian Symposium on Human Factors in Computing Systems (IHC'16), 2016.

[4] F. C. Mendonça, I. Gasparini, R. Schroeder, M. S. Silveira, S. D. J. Barbosa. Scientific Collaboration Networks of the Academic Brazilian Community of HCI. In: Brazilian Symposium on Human Factors in Computing Systems, 2018.

[5] A. Tramontin, R. Sohn, B. Oliveira, R. Pereira, I. Gasparini. Emotion and Movement with AppIHC: Promoting Interaction and Socialization Among Participants of Scientific Events via Mobile Application. In: International Conference on Human-Computer Interaction (HCII), 2019, Orlando. Springer, 2019. v. 11567. p. $62-75$.

[6] C. S. de Borba, I. Gasparini. Quais as Melhores Maneiras de Apresentar as Recomendações para os Usuários? Um Mapeamento Sistemático da Literatura. iSys - Revista Brasileira de Sistemas de Informação (to appear). 